\title{
Impact of Acute Kidney Injury on Chronic Kidney Disease and Its Progression
}

\author{
Scott Sanoff · Mark D. Okusa
}

Departments of Medicine and Pharmacology and Center for Immunity, Inflammation and Regenerative Medicine, University of Virginia, Charlottesville, Va., USA

\begin{abstract}
Acute kidney injury (AKI) is a devastating clinical problem that affects a growing number of patients, especially elderly ones, and is associated with high morbidity and mortality. It was previously thought that patients who survive an episode of AKI recover renal function without further sequelae; however, recent population-based studies suggest that this may not be the case. New clinical studies suggest that a strikingly large percentage of patients who have AKI do not fully recover renal function or require permanent renal replacement therapy, and that this population has an important impact on the epidemiology of chronic kidney disease (CKD) and end-stage renal disease. These clinical studies verify animal studies that have established a link between AKI and CKD progression. Future clinical studies are underway to prospectively characterize the natural history of AKI and CKD progression and to identify predictive biomarkers.
\end{abstract}

Copyright $\odot 2011$ S. Karger AG, Basel

Recent studies suggest that there is an increase in the contribution of acute kidney injury (AKI) to incident and prevalent chronic kidney disease (CKD) and end-stage renal disease (ESRD). In the USA, approximately 10-40 million adults have CKD and 527,283 patients were treated for ESRD (with dialysis or kidney transplantation) in 2007 [1], a number which is projected to increase to 0.8 million by 2020 . There is also evidence that the incidence of AKI is rising. Over the past 10-15 years, there has been an impressive increase in the incidence of AKI $[2,3]$ that appears to be related to the increase in invasive medical and surgical procedures, particularly in the aggressive management of critically ill elderly patients. In critically ill patients, mortality is $40-60 \%$ [4-7] and is traditionally attributed to comorbid conditions. Among these patients, AKI has been shown to have an independent negative association with mortality $[8,9]$. Even a small rise in serum creatinine of $0.3-0.4 \mathrm{mg} /$ $\mathrm{dl}$ has been associated with 1.7 times the odds of dying (multivariable OR 1.7; 95\% CI 1.2-2.6) [10]. The trend in mortality appears to be changing. Although mortality may be declining, this may lead to a greater burden of ESRD or CKD. Patients who survive AKI may have several different outcomes: (1) full recovery; (2) development of AKI leading to CKD; (3) exacerbation of preexisting $\mathrm{CKD}$ leading to more advanced CKD or ESRD; (4) non-recovery of AKI leading to ESRD. This review summarizes the most recent reports on the relationship between AKI and CKD/ESRD. 
Acute Kidney Injury Associated with Increased Risk of Chronic Kidney Disease and End-Stage Renal Disease

Several factors taken together suggest that AKI is an important contributor to CKD and ESRD. Using the Nationwide Inpatient Sample, a nationally representative sample of discharges from acute-care nonfederal hospitals, Waikar et al. [2] found that the incidence of AKI not requiring and requiring dialysis (per 100,000 population) rose from 61 to 288 and 4 to 27 , respectively, between 1988 and 2002. The hospital mortality in patients with AKI declined steadily (from 40.4 to $20.3 \%$; $\mathrm{p}<0.001$ ). These results suggest that more patients are suffering from AKI, but fewer are dying. In those patients surviving AKI, progressive CKD and ESRD may ensue. Despite the increase in the incident AKI population, there is evidence suggesting that the prevalent CKD stage 3 and 4 population changed very little over time. Hsu [25] recently analyzed data from the National Health and Nutrition Examination Survey (NHANES) on the prevalent CKD population and incident AKI population estimates from 1988 to 2002 [11]. The calculated incidence of ESRD from AKI survivors (per 100,000 population) increased from 0.4 to 4.9 (an absolute rate difference of 4.5 per 100,000 population) and the incidence of ESRD from USRDS increased from 16 to 34 (an absolute rate difference of 18 per 100,000 population). Thus, the potential fraction of the increase in ESRD incidence attributable to AKI was estimated to be $25 \%$.

Ishani et al. [26] used a 5\% random sample of Medicare beneficiary claims from Centers for Medicare and Medicaid Services and the ESRD database from USRDS, and studied a cohort of 233,803 patients that were hospitalized in 2000 with a discharge diagnosis of AKI. The likelihood of initiating ESRD treatment after AKI alone was significantly greater than for those with no AKI and no CKD, or those with CKD alone. These results suggest a potential role for AKI as a predisposing factor to ESRD.

Evidence for the important contribution of AKI to CKD/ESRD is not limited to adults. In a recent study, $>50 \%$ of pediatric patients showed evidence of progressive kidney injury and hypertension within 3-5 years of an initial episode of AKI [12]. Longer-term mortality rates were also increased.

In summary, population-based evidence strongly suggests an important and growing role of AKI in the epidemiology of CKD and ESRD.

\section{Clinical Evidence That Acute Kidney Injury Leads to Chronic Kidney Disease}

In a recent systematic review of the literature between 1985 and 2007, Coca et al. [27] described 8 studies that contained over 47,000 participants and found that the incidence rate of CKD after an episode of AKI was 7.8 events/100 patient-years, and the rate of ESRD was 4.9 events 100 patientyears. If one excludes preexisting $\mathrm{CKD}$, the rate of CKD after an episode of AKI was 4.2 events/100 patient-years. This large review provides strong support to the growing body of literature linking AKI to CKD.

In a province-wide sample of approx. 930,000 adult Canadians, James et al. [13] assessed hospital admission with acute kidney injury, mortality, and CKD/ESRD. They found that the risk of progression to ESRD varied and increased depending on the eGFR and degree of proteinuria. During a follow-up period of 35 months (range 0-59 months), they found that the risk of subsequent adverse outcomes including death and ESRD varied with eGFR and proteinuria. Thus GFR, AKI, and proteinuria serve as prognostic indicators for incident ESRD.

To address the impact of dialysis during an episode of AKI on CKD and ESRD, Wald [28] recently published data from a population-based cohort study of patients in Ontario, Canada, with AKI who required in-hospital dialysis and survived free of dialysis for at least 30 days after discharge. This study examined 3,769 adults with AKI requiring in-hospital dialysis and 13,598 matched controls that did not have AKI or did not receive dialysis during their hospitalization. In the group of patients with AKI requiring 
dialysis, the incidence rate of patients requiring chronic dialysis was 2.63 per 100 person-years and for the control group the incidence rate was 0.91 per 100 person-years. These results indicate that in-hospital dialysis during AKI was associated with an increased risk of chronic dialysis consistent with other studies $[14,15]$.

There is evidence of a long-term decline in kidney function following coronary angiography. James et al. [16] assessed changes in kidney function over a 3-year period following adults undergoing coronary angiography. Compared to patients without AKI, the odds of a sustained decline in kidney function at 3 months following angiography increased more than 4-fold for patients with mild (50-59\% or $\geq 0.3 \mathrm{mg} /$ dl) to more than 17 -fold for those with moderate or severe $(\geq 100 \%)$ acute kidney injury. Following mild or moderate-severe AKI, among the patients with eGFR less than $90 \mathrm{ml} / \mathrm{min}$ per $1.73 \mathrm{~m}^{2}$, the rates of decline in GFR were 0.2 and $2.8 \mathrm{ml} / \mathrm{min}$, respectively. These results indicate that angiography is associated with a decline in kidney function.

In a study of 556,090 adult members of Kaiser Permanente of Northern California hospitalized with baseline GFR of $\geq 45 \mathrm{ml} / \mathrm{min} / 1.73 \mathrm{~m}^{2}$, and who survived hospitalization, dialysis-requiring acute renal failure was independently associated with a 28-fold increase in the risk of developing stage 4 or 5 CKD [17].

These studies carried out over the past few years strongly support the association between $\mathrm{AKI}$ and CKD/ESRD, whether the severity of AKI is mild or severe. The important question to be answered is whether or not there is a causal relation between AKI and CKD. Furthermore, as a corollary, does avoiding transient elevations in serum creatinine prevent AKI? The vast majority of these studies have focused on clinical outcomes following hospitalization for AKI using administrative databases that capture widely different populations and clinical settings. Thus, although substantial studies have reported a correlation between AKI and increased risk of CKD and ESRD, these studies have several limitations. First, the retrospective and observational nature of prior studies make their data vulnerable to unavoidable bias and confounding variables, precluding any assessment of cause and effect between AKI and CKD. Second, many of these studies lack of rigorous quantification of baseline renal function. By definition, AKI occurrence in hospital requires a change in serum creatinine from 'baseline'. Often times there is no baseline serum creatinine, and thus the use of an imputed estimate of kidney function has led to misclassification of AKI. Many of these studies relied on diagnostic codes for AKI in defining AKI and presence and absence of CKD. Third, it may be that patients with AKI that progressed to CKD/ ESRD may have underlying comorbidities that put them at high risk of progressive deterioration of renal function following AKI. Alternatively, it maybe that in some fashion the injured kidney initiated a pathophysiological process leading to progressive kidney fibrosis and ultimately to failure. There is considerable animal data suggesting that this may indeed be the case, but this has yet to be determined.

\section{Biomarkers to Describe and Predict the Development of Chronic Kidney Disease Following Acute Kidney Injury}

The discovery and growing number of investigations of biomarkers in AKI offer the promise of new tools to help predict and describe the transition to CKD following AKI, and to monitor the success of available therapies. Among the best-described biomarkers of AKI are neutrophil gelatinase-associated lipocalin (NGAL), kidney injury molecule 1 (KIM1), and liver-type fattyacid-binding protein (L-FABP). These are perhaps best known for their sensitivity as early markers of AKI, as each is detectable in serum and/or urine within hours of AKI, prior to a discernable rise in serum creatinine [18-20]. In addition to their role as markers of early AKI, each has been linked in humans to more indolent forms of chronic kidney disease at various stages [21]. For example, NGAL was found to be elevated in a group of pediatric patients with type 1 diabetes 
(vs. healthy controls), prior to the development of significant proteinuria or creatinine elevation, but still demonstrated a positive correlation with urinary albumin excretion, suggesting NGAL may be a sensitive marker and predictor of early CKD [22]. Biomarkers have also been shown to have prognostic value in CKD. For example, a single 'baseline' measurement of NGAL was shown at follow-up to be an independent predictor of later CKD progression among individuals affected by CKD of various etiologies and stages [23]. Moreover, in animal models of AKI, serial measurements of NGAL and KIM1 following ischemic injury reveal elevations throughout the late repair phase of AKI, suggesting that these molecules may indicate progression to CKD following AKI. Further, their elevation also correlates with progressive renal fibrosis, suggesting these molecules may play a functional role in the development of CKD, offering potential insight into this process.

Despite the robust literature describing serum and urinary biomarkers in the early course of $\mathrm{AKI}$ and $\mathrm{CKD}$, and the animal data linking AKI and $\mathrm{CKD}$, there is a paucity of published prospective longitudinal data in humans examining biomarkers in the transition from AKI to CKD. The Assessment, Serial Evaluation and Subsequent Sequelae of Acute Kidney Injury (ASSSESS-AKI) Consortium, established in 2008 by NIH to prospectively compare the differences in renal and cardiovascular outcomes and death in patients with AKI is likely to contribute significantly in this regard [24]. These investigators plan to enroll a prospective cohort of 1,100 adult subjects with AKI and 100 children undergoing cardiac surgery and followed for 4 years to characterize the natural history of AKI. In particular, they will examine the effect of a hospitalized episode of
AKI on the risk of developing future CKD and ESRD. The utility of urine and blood biomarkers for identifying the high-risk patient who is destined to be subjected to progressive decline in renal function will be assessed in this study. Markers of interest include IL-18, Kim 1, NGAL, cystatin C, and LFABP. Identifying those patients at risk prospectively with biomarkers may allow aggressive interventions to delay or halt the progression of kidney disease.

\section{Conclusion}

There is accumulating evidence to suggest that in some cases patients who survive hospital-acquired AKI may ultimately develop progressive kidney disease leading to ESRD. However, it is important to realize that these studies do not establish cause and effect. Thus, in the future it is imperative that we determine the causal relation between AKI and CKD/ESRD as well as the pathogenic mechanisms responsible for the decline in kidney function after AKI. Prevention of AKI by identifying high-risk patients, providing adequate hydration, and discontinuing potentially harmful drugs will be important in the short- and long-term outcomes of these patients. Furthermore, a better understanding of the pathogenic mechanisms of AKI and CKD progression may lead to specific therapies to block this process.

\section{Acknowledgement}

The authors gratefully acknowledge support by NIH grants R01DK08525 R01 DK083406 and R01 DK062324.

\section{References}

1 United States Renal Data System: Annual Data Report 2009: Atlas of Chronic Kidney Disease and EndStage Renal Disease in the United States. Minneapolis, USRDS, 2009.

\footnotetext{
2 Waikar SS, Curhan GC, Wald R, et al: Declining mortality in patients with acute renal failure, 1988 to 2002 . J Am Soc Nephrol 2006;17:1143-1150.
} 
4 Uchino S, Kellum JA, Bellomo R, et al: Acute renal failure in critically ill patients: a multinational, multicenter study. JAMA 2005;294:813-818.

5 Bellomo R, Ronco C, Kellum JA, et al: Acute renal failure - definition, outcome measures, animal models, fluid therapy and information technology needs: the Second International Consensus Conference of the Acute Dialysis Quality Initiative (ADQI) Group. Crit Care 2004;8:R204-R212.

6 Liano F, Pascual J: Outcomes in acute renal failure. Semin Nephrol 1998;18 541-550.

7 Hoste EA, Schurgers M: Epidemiology of acute kidney injury: how big is the problem? Crit Care Med 2008;36:S146-S151.

$>8$ Chertow GM, Levy EM, Hammermeister KE, et al: Independent association between acute renal failure and mortality following cardiac surgery. Am J Med 1998;104:343-348.

-9 Levy EM, Viscoli CM, Horwitz RI: The effect of acute renal failure on mortality: a cohort analysis. JAMA 1996;275:1489-1494.

10 Chertow GM, Burdick E, Honour M, et al: Acute kidney injury, mortality, length of stay, and costs in hospitalized patients. J Am Soc Nephrol 2005; 16:3365-3370.

11 Coresh J, Selvin E, Stevens LA, et al: Prevalence of chronic kidney disease in the United States. JAMA 2007;298: 2038-2047.

12 Askenazi DJ, Feig DI, Graham NM, et al: 3-5 year longitudinal follow-up of pediatric patients after acute renal failure. Kidney Int 2006;69:184-189.
13 James MT, Hemmelgarn BR, Wiebe $\mathrm{N}$, et al: Glomerular filtration rate, proteinuria, and the incidence and consequences of acute kidney injury: a cohort study. Lancet 2010;376: 2096-2103.

14 Bagshaw SM, Laupland KB, Doig CJ, et al: Prognosis for long-term survival and renal recovery in critically ill patients with severe acute renal failure: a population-based study. Crit Care 2005;9:R700-R709.

15 Morgera S, Kraft AK, Siebert G, et al: Long-term outcomes in acute renal failure patients treated with continuous renal replacement therapies. Am J Kidney Dis 2002;40:275-279.

16 James MT, Ghali WA, Tonelli M, et al: Acute kidney injury following coronary angiography is associated with a long-term decline in kidney function. Kidney Int 2010;78:803-809.

17 Lo LJ, Go AS, Chertow GM, et al: Dialysis-requiring acute renal failure increases the risk of progressive chronic kidney disease. Kidney Int 2009;76:893-899.

18 Wagener G, Gubitosa G, Wang S, et al: Urinary neutrophil gelatinaseassociated lipocalin and acute kidney injury after cardiac surgery. Am J Kidney Dis 2008;52:425-433.

19 Han WK, Waikar SS, Johnson A, et al: Urinary biomarkers in the early diagnosis of acute kidney injury. Kidney Int 2008;73:863-869.

20 Portilla D, Dent C, Sugaya T, et al: Liver fatty acid-binding protein as a biomarker of acute kidney injury after cardiac surgery. Kidney Int 2008;73:465-472.

21 Goldstein SL, Devarajan P: Acute kidney injury in childhood: should we be worried about progression to CKD? Pediatr Nephrol 2011;26:509-522.
22 Zachwieja J, Soltysiak J, Fichna P, et al: Normal-range albuminuria does not exclude nephropathy in diabetic children. Pediatr Nephrol 2010;25: 1445-1451.

23 Bolignano D, Lacquaniti A, Coppolino G, et al: Neutrophil gelatinase-associated lipocalin (NGAL) and progression of chronic kidney disease. Clin J Am Soc Nephrol 2009;4:337-344.

24 Go AS, Parikh CR, Ikizler TA, et al: The assessment, serial evaluation, and subsequent sequelae of acute kidney injury (ASSESS-AKI) study: design and methods. BMC Nephrol 2010;11:22.

25 Hsu CY: Linking the population epidemiology of acute renal failure, chronic kidney disease and end-stage renal disease. Curr Opin Nephrol Hypertens 2007;16:221-226.

26 Ishani A, Xue JL, Himmelfarb J, Eggers PW, Kimmel PL, Molitoris BA, Collins AJ: Acute kidney injury increases risk of ESRD among elderly. J Am Soc Nephrol 2009;20:223-228.

27 Coca SG, Yusuf B, Shlipak MG, Garg AX, Parikh CR: Long-term risk of mortality and other adverse outcomes after acute kidney injury: a systematic review and meta-analysis. Am J Kidney Dis 2009;53:961-973.

28 Wald R, Quinn RR, Luo J, Li P, Scales DC, Mamdani MM, Ray JG, University of Toronto Acute Kidney Injury Research Group: Chronic dialysis and death among survivors of acute kidney injury requiring dialysis. JAMA 2009;302:1179-1185, erratum in JAMA 2009;302:1532.
Mark D. Okusa, MD

Division of Nephrology, Box 800133

University of Virginia Health System

Charlottesville, VA 22908 (USA)

Tel. +1 434924 2187, E-Mail mdo7y@virginia.edu 